\title{
A conceptual database design for the operation of West Africa Examination Council (WAEC) Co-Operative Credit Union
}

\author{
Gabriel Kofi Armah \\ Gabrielarmah1@yahoo.com \\ University for Development Studies (Faculty of Mathematical Sciences)
}

\begin{abstract}
In this paper, we present the conceptual database design for the WAEC co-operative credit union operation.. The Entity -Relationship model is employed to come out with the various entities, attributes, and relationships between entities.
\end{abstract}

\section{INTRODUCTION}

Database design focuses on the design of databases and files to be used by a proposed information system. Database design involves three main levels of design: conceptual, logical and physical design. In this paper our focus is on the conceptual database design; This is the process of constructing a model of the information used in an enterprise, independent of all physical consideration, i.e. both software and hardware (Connolly and Begg, 1998). The following steps are involved in creating the WAEC conceptual database design:

This entails examining the requirement specification for the user's particular function within the enterprise to identify the entities. Entities are presented in the form of nouns or noun phrase. Objects such as people, places or concept (excluding those which are mere attributes) are identified as entities. Having identified the entity types, the next step is to identify relationship types. Once again the requirement specification was consulted. Relationships are normally presented in the form of verbs or verb expressions. As relationship types are identified, the cardinality and participation constraints are determined. The cardinality can be 1:1, 1: M or M: $\mathrm{N}$ and the participation can be either total or partial. The relationships are documented and given names which are meaningful to the user.

The final step is the identification of the attributes associated with each entity or relationship type.

\section{IDENTIFICATION}

(a) ENTITY TYPES

An entity type is an object or concept that is identified by the enterprise as having an independent existence. The entities identified in the requirements specification are: member contribution,loan, dividend,staff, withdrawal,loanrepayment, hirepurchase, interest, next-of-kin and bank-account.

\section{(b) RELATIONSHIP TYPES}

A relationship type is a meaningful association among entity types.

The next step is the identification of the relationships between the identified entities in section $2 a$. The major relationships identified in the requirement specification alongside with their participation constraints and cardinality ratios are tabulated in table $2 \mathrm{~b}$ below: 
Am. J. Sci. Ind. Res., 2011, 2(6): 846-851

Table 2b;

\begin{tabular}{|c|c|c|c|c|}
\hline \multirow{2}{*}{ Entity Type } & Relationship Type & Entity Type & Cardinality ratio & Participation \\
\hline Member & Pays & Contribution & $1: M$ & T:T \\
& Relates & Next-Of-Kin & T:T & P:T \\
& BuysOn & Wirepurchase & $1: M$ & P:T \\
& Makes & Dithdrawal & $1: M$ & T:T \\
\hline Loan & Given & Member & $1: 1$ & T:P \\
& GrantedTo & Member & $1: M$ & T:T \\
\hline Loan-Repayment & Attracts & Interest & $1: M$ & T:P \\
\hline
\end{tabular}

"P" represents partial participation and "T" represents total participation

\section{(c) ATTRIBUTES}

An attribute is a property of an entity or a relationship type.Having identified the entities and relationships the next step is to identify all the attributes associated with the various entities and relationships (if any). In identifying the entities, the description of each is given, stating its type, size, whether it is a primary key, whether it can contain null values and whether or not it is derived. This is documented in the data dictionary, as shown in table $\mathbf{2 c}$ below.

\begin{tabular}{|c|c|c|c|c|c|c|}
\hline Entity & $\begin{array}{l}\text { Names of } \\
\text { Attributes }\end{array}$ & Description & $\begin{array}{l}\text { Data Type } \\
\text { and Length }\end{array}$ & Constraints & $\begin{array}{l}\text { Null Value } \\
\text { [Yes or No] }\end{array}$ & Derived \\
\hline Member & $\begin{array}{l}\text { MemberRegNum } \\
\text { Fname } \\
\text { Lname } \\
\text { Address } \\
\text { Telnum } \\
\text { Sex } \\
\text { DOB } \\
\text { Age } \\
\text { Rank }\end{array}$ & $\begin{array}{l}\text { First Name } \\
\text { Last name }\end{array}$ & \begin{tabular}{l}
14 variable \\
characters \\
20 variable \\
characters \\
20 variable \\
characters \\
50 variable \\
characters \\
14 variable \\
characters 6 \\
fixed \\
characters \\
\multicolumn{1}{c}{ Date } \\
Integer \\
20 variable \\
characters
\end{tabular} & Primary key & $\begin{array}{l}\text { No } \\
\text { No } \\
\text { No } \\
\text { No } \\
\text { No } \\
\text { No } \\
\text { No } \\
\text { No } \\
\text { No }\end{array}$ & $\begin{array}{l}\text { No } \\
\text { No } \\
\text { No } \\
\text { No } \\
\text { Yes } \\
\text { No } \\
\text { No } \\
\text { Yes } \\
\text { No }\end{array}$ \\
\hline
\end{tabular}

Attribute, Data Types and Constraints

Table 2c.1

\begin{tabular}{|l|l|l|l|l|l|l|}
\hline Entity & $\begin{array}{l}\text { Names Of } \\
\text { Attributes }\end{array}$ & Description & $\begin{array}{l}\text { Data Type } \\
\text { and Length }\end{array}$ & Constraint & $\begin{array}{l}\text { Null value } \\
\text { [Yes or No] }\end{array}$ & Derived \\
\hline Loan & Loannum & Loan number & $\begin{array}{l}14 \text { fixed } \\
\text { characters }\end{array}$ & Primary key & No & No \\
& $\begin{array}{l}\text { MemberregNum } \\
\text { Amount }\end{array}$ & $\begin{array}{l}\text { Integer } \\
\text { Integer }\end{array}$ & & No & No \\
& Instalment & instalment & $\begin{array}{l}\text { Integer } \\
\text { Inter }\end{array}$ & & No & No \\
& & & & No & No \\
& Startdate & & Date & & No \\
& Enddate & & Date & & No \\
\hline
\end{tabular}


Am. J. Sci. Ind. Res., 2011, 2(6): 846-851

\begin{tabular}{|l|l|l|l|l|l|l|}
\hline Bank-Account & Accountnum & $\begin{array}{l}\text { Account } \\
\text { number }\end{array}$ & $\begin{array}{l}\text { Integer } \\
40 \text { variable } \\
\text { characters } \\
30 \text { variable } \\
\text { characters } \\
20 \text { variable } \\
\text { characters } \\
\text { Bccountname }\end{array}$ & Alternate key & No & No \\
& Thal & Total balance & Number & No Ko & No \\
\hline
\end{tabular}

Attribute, Data Types and Constraints

Table 2c.2

\begin{tabular}{|c|c|c|c|c|c|c|}
\hline Entity & $\begin{array}{l}\text { Name Of } \\
\text { Attributes }\end{array}$ & Description & $\begin{array}{l}\text { Data type and } \\
\text { Length }\end{array}$ & Constraint & $\begin{array}{l}\text { Null value [Yes } \\
\text { or No ] }\end{array}$ & Derived \\
\hline $\begin{array}{l}\text { Next-Of- } \\
\text { kin }\end{array}$ & $\begin{array}{l}\text { MemberRegNum } \\
\text { NextOfkinNum } \\
\text { Relationship }\end{array}$ & & $\begin{array}{l}\text { Integer } \\
\text { Integer } \\
20 \text { variable } \\
\text { characters }\end{array}$ & & $\begin{array}{l}\text { No } \\
\text { No } \\
\text { No }\end{array}$ & $\begin{array}{l}\text { No } \\
\text { No } \\
\text { No }\end{array}$ \\
\hline
\end{tabular}

Table 2c.3

\begin{tabular}{|c|c|c|c|c|c|c|}
\hline Entity & $\begin{array}{l}\text { Name of } \\
\text { Attributes }\end{array}$ & Description & $\begin{array}{l}\text { Data type and } \\
\text { Length }\end{array}$ & Constraint & $\begin{array}{l}\text { Null value [Yes } \\
\text { or No ] }\end{array}$ & Derived \\
\hline Officer & $\begin{array}{l}\text { MemberRegNum } \\
\text { Fname } \\
\text { Lname } \\
\text { Address } \\
\text { Telnum } \\
\text { Sex } \\
\text { DOB } \\
\text { Age } \\
\text { Rank }\end{array}$ & $\begin{array}{l}\text { Executive of } \\
\text { union } \\
\text { First Name } \\
\text { Last name } \\
\text { Date of birth }\end{array}$ & $\begin{array}{l}14 \text { variable } \\
\text { characters } \\
20 \text { variable } \\
\text { characters } \\
20 \text { variable } \\
\text { characters } \\
50 \text { variable } \\
\text { characters } \\
14 \text { variable } \\
\text { characters } \\
6 \text { fixed } \\
\text { characters } \\
\text { Date } \\
\text { Integer } \\
20 \text { variable } \\
\text { characters }\end{array}$ & Primary key & $\begin{array}{l}\text { No } \\
\text { No } \\
\text { No } \\
\text { No } \\
\text { No } \\
\text { No } \\
\text { No } \\
\text { No } \\
\text { No }\end{array}$ & $\begin{array}{l}\text { No } \\
\text { No } \\
\text { No } \\
\text { No } \\
\text { Yes } \\
\text { No } \\
\text { No } \\
\text { Yes } \\
\text { No }\end{array}$ \\
\hline
\end{tabular}

Attribute, Data Types and Constraints

Table 2c.4

\begin{tabular}{|l|l|l|l|l|l|l|}
\hline Entity & $\begin{array}{l}\text { Name Of } \\
\text { Attributes }\end{array}$ & Description & $\begin{array}{l}\text { Data type } \\
\text { and Length }\end{array}$ & Constraint & $\begin{array}{l}\text { Null value } \\
\text { [Yes or No ] }\end{array}$ & Derived \\
\hline Loanrepayment & Amount & Total balance & $\begin{array}{l}\text { Integer } \\
\text { Integer }\end{array}$ & & No & No \\
& Tbal & Start Date & Date & & No \\
& Sdate & End Date & Nate & & No \\
& Edate & No & & No \\
\hline
\end{tabular}

Attribute, Data Types and Constraints 
Am. J. Sci. Ind. Res., 2011, 2(6): 846-851

Table 2c.5

\begin{tabular}{|c|c|c|c|c|c|}
\hline Dividend & $\begin{array}{l}\text { Amount } \\
\text { Date } \\
\text { MemberRegNum }\end{array}$ & & $\begin{array}{l}\text { Integer } \\
\text { Date } \\
\text { Integer }\end{array}$ & $\begin{array}{l}\text { Yes } \\
\text { No } \\
\text { No }\end{array}$ & $\begin{array}{l}\text { Yes } \\
\text { No } \\
\text { No }\end{array}$ \\
\hline Interest & $\begin{array}{l}\text { Percentage } \\
\text { Loannum } \\
\text { Period } \\
\text { Form } \\
\text { InterestNum }\end{array}$ & $\begin{array}{l}\text { Normally } 5 \% \\
\text { Simple or } \\
\text { compound } \\
\text { interest }\end{array}$ & $\begin{array}{l}\text { Integer } \\
\text { Integer } \\
\text { Dater } \\
15 \text { variable } \\
\text { characters }\end{array}$ & $\begin{array}{l}\text { No } \\
\text { No } \\
\text { No } \\
\text { No } \\
\\
\text { No }\end{array}$ & $\begin{array}{l}\text { No } \\
\text { No } \\
\text { No } \\
\text { No } \\
\\
\text { No }\end{array}$ \\
\hline
\end{tabular}

Attribute, Data Types and Constraints

Table 2c.6

\begin{tabular}{|c|c|c|c|c|c|}
\hline Hirepurchase & $\begin{array}{l}\text { Itemname } \\
\text { HirepurchaseNum } \\
\text { Quantity } \\
\text { MemberRegNum } \\
\text { Tamount } \\
\text { Instalment } \\
\text { Tbal }\end{array}$ & $\begin{array}{l}\text { Total amount } \\
\text { Total balance }\end{array}$ & $\begin{array}{l}40 \text { variable } \\
\text { characters } \\
\text { Integer } \\
\text { Integer } \\
\text { Integer } \\
\text { Integer } \\
\text { Integer } \\
\text { Integer }\end{array}$ & $\begin{array}{l}\text { No } \\
\text { No } \\
\text { No } \\
\text { No } \\
\text { No } \\
\text { No } \\
\text { No }\end{array}$ & $\begin{array}{l}\text { No } \\
\text { No } \\
\text { No } \\
\text { No } \\
\text { No } \\
\text { No } \\
\text { No }\end{array}$ \\
\hline Contribution & $\begin{array}{l}\text { Amount } \\
\text { Date } \\
\text { CurrentBal } \\
\text { ContrbutionNum } \\
\text { MemberRegNum }\end{array}$ & $\begin{array}{l}\text { Savings } \\
\text { Balance } \\
\text { Amount } \\
\text { contributed per } \\
\text { month per } \\
\text { member }\end{array}$ & $\begin{array}{l}\text { Integer } \\
\text { Date } \\
\text { Integer } \\
\text { Integer } \\
\text { Integer }\end{array}$ & $\begin{array}{l}\text { No } \\
\text { No } \\
\text { No } \\
\text { No } \\
\text { No }\end{array}$ & $\begin{array}{l}\text { No } \\
\text { No } \\
\text { No } \\
\text { No } \\
\text { No }\end{array}$ \\
\hline Withdrawal & $\begin{array}{l}\text { Staffnum } \\
\text { Date } \\
\text { Reason } \\
\text { Accruals }\end{array}$ & & $\begin{array}{l}\text { Integer } \\
\text { Date } \\
30 \text { variable } \\
\text { characters } \\
\text { Integer }\end{array}$ & $\begin{array}{l}\text { No } \\
\text { No } \\
\text { No } \\
\text { No }\end{array}$ & $\begin{array}{l}\text { No } \\
\text { No } \\
\text { No } \\
\text { No }\end{array}$ \\
\hline
\end{tabular}

Attribute, Data Types and Constraints 
Am. J. Sci. Ind. Res., 2011, 2(6): 846-851

\subsection{A DIAGRAMMATIC REPRESENTATION OF THE WAEC ER-MODEL}

Having gone through the above steps, the conceptual entity relation diagram is then drawn. This is shown in figure 3.1.

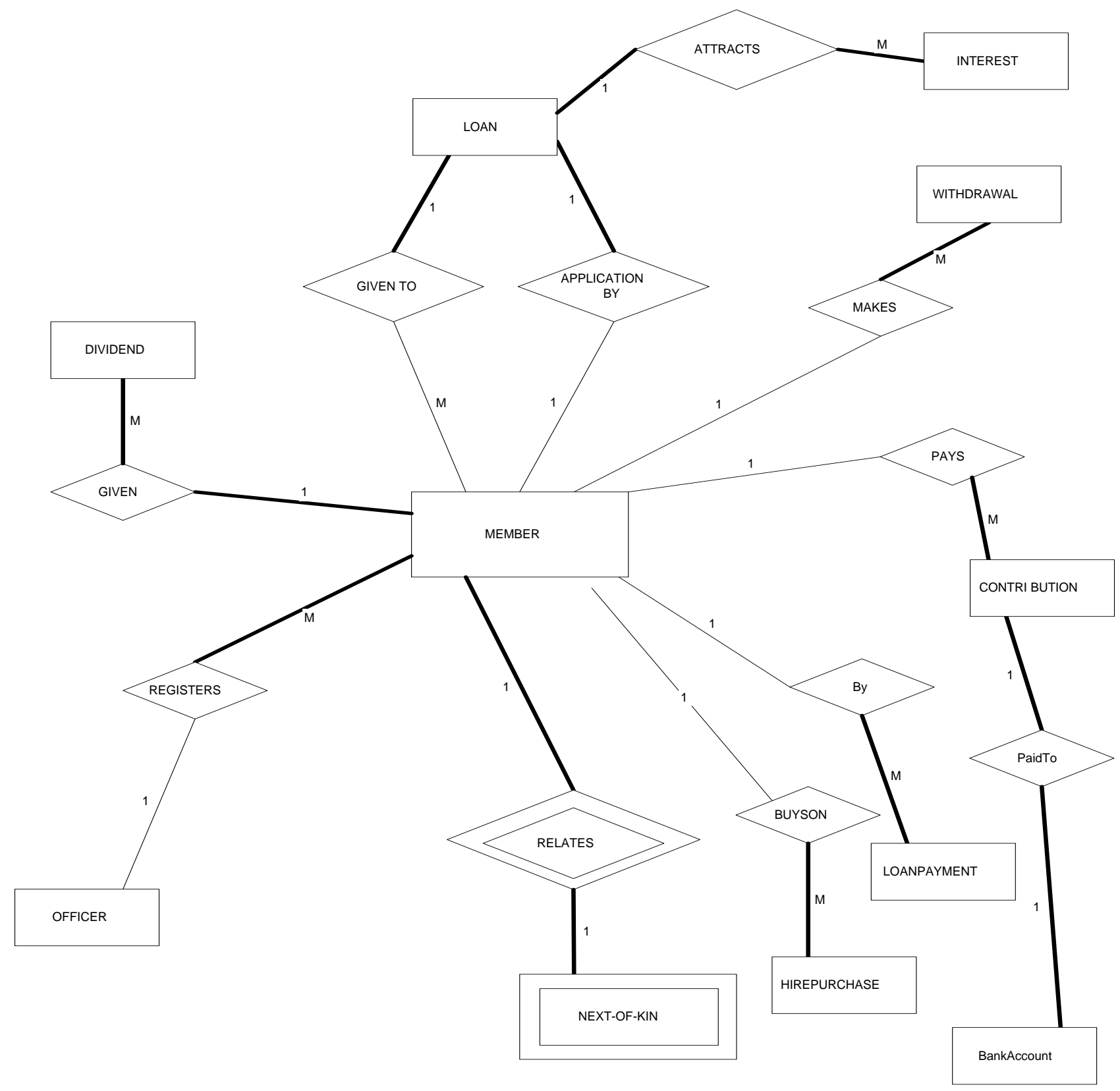

FIG. 3.1 A Sketch of WAEC CU Conceptual Database Model 


\section{CONCLUSION}

A conceptual model of a computerized information system for the operations of West Africa examination council (WAEC) co-operative credit union has been designed.

We employ the entity-relationship model to depict the WAEC co-operative credit union entities and the relationships existing between them.

This is the first of a three -part design of a database. In subsequent work we shall talk about the logical and the physical designs.

\section{REFERENCES}

1 Laudon, Kenneth C. \& Jane P. Laudon, "Management Information System" $4^{\text {th }}$ Ed., Prentice-Hall, New Delhi, India 1999 pp 378, 412-449

2. Hicks, J.O.(jnr.) ,"Management Information Systems". A User Perspective, West Publishing Company, USA, pp, 426-432

3. Date,C.J., "An Introduction To Database Systems", 1995, p.29

4. Connolly, Thomas M. \& Carolyn E. Begg, "Database Systems, A Practical to Design, Implementation, and Management", $2^{\text {nd }}$ Edition, Addison-Wesley Publishers USA 1999, pp. 6-229.

5.Peter Rob \&Caros Coronel, Database Systems: Design, Implementation, \& Management, $4^{\text {th }}$ Edition, Thomson Learning,pp188-189. 